\title{
Gestational Trophoblastic Neoplasia: A Retrospective Study of 23 Cases
}

\author{
Rajendra Kumar Tanwar ${ }^{1, ~ *, ~ B h a r t i ~ S a x e n a ~}{ }^{2}$, Radha Garg $^{2}$, Harsh Goyal$^{1}$, Des Deepak ${ }^{3}$ \\ ${ }^{1}$ Deaprtment of Radiation Oncology, Government Medical College, Kota, Rajasthan University of Health Sciences, Jaipur, Rajasthan, India \\ ${ }^{2}$ Deaprtment of Obstetrics and Gynecology, Government Medical College, Kota, Rajasthan University of Health Sciences, Jaipur, Rajasthan, \\ India \\ ${ }^{3}$ Department of Obstetrics and Gynecology, Rajdeep Fertility Research Centre, Kota, Rajasthan, India
}

\section{Email address:}

rktanwar@rediffmail.com (R. K. Tanwar), bharti.saxena.kota@gmail.com (B. Saxena), radha9818020671@gmail.com (R. Garg), drharsh222@gmail.com (H. Goyal),drdeshdeepak2013@gmail.com (D. Deepak)

${ }^{*}$ Corresponding author

\section{To cite this article:}

Rajendra Kumar Tanwar, Bharti Saxena, Radha Garg, Harsh Goyal, Des Deepak. Gestational Trophoblastic Neoplasia: A Retrospective Study of 23 Cases. Cancer Research Journal. Vol. 8, No. 3, 2020, pp. 45-50. doi: 10.11648/j.crj.20200803.11

Received: July 13, 2020 Accepted: August 3, 2020; Published: August 10, 2020

\begin{abstract}
Background - The aim of this study is to analyze the clinicopathological features, diagnosis and appropriate treatment outcome of patients with gestational trophoblastic neoplasia (GTN), a heterogenous group of disorder in a government tertiary care hospital center. Method- This is a retrospective study of 23 patients from $2002-2020$ over 18 years. The patients were evaluated on the basis of their age, obstetric history and the treatment received. All the patients were scored according to Modified World Health Organization (WHO) prognostic scoring system as adapted by FIGO Year 2000. Low risk group patients were given injection Methotrexate + Calcium Leucovorin and high risk group were given EMACO regimen. After treatment, the patient follow up was till date or minimum up to 2 years. The response to treatment was evaluated by regular clinical and radiological examination and serum $\beta$-hCG level estimation. Results - Three out of 23 patients who relapsed after primary chemotherapy were given second line chemotherapy. All three patients achieved complete remission (CR) after salvage chemotherapy. Thus overall response rate is $100 \%$. Conclusion - Proper diagnosis, close monitoring and follow up with $\beta$-hCG value is of utmost importance in the management of GTN, WHO/FIGO scoring should be done and managed with chemotherapy treatment according to the risk assessment.
\end{abstract}

Keywords: Gestational Trophoblastic Neoplasia, EMACO Regimen, Invasive Mole, Choriocarcinoma

\section{Introduction}

Gestational trophoblastic disease (GTD) is a spectrum of rare conditions that arise due to abnormal proliferation of trophoblastic cells in placental microvilli. They consist of Vesicular Mole (Complete and Partial), which are benign lesions that resolve following uterine evacuation in majority of cases; [1, 2] GTN [invasive mole, Choriocarcinoma (CCA), placental site trophoblastic tumor (PSTT) and epethelioid trophoblastic tumors (ETT)] most commonly arise after a molar pregnancy, although they can develop after any pregnancy. The incidence of gestational CCA is $<1 \%$ of all malignancies in women. Approximately $15 \%$ of complete hydatidiform mole will result in local invasion (Invasive mole) and 5\% will develop metastasis (CCA) to lungs or vagina. Partial hydatidiform mole occurs in 3-5\% of molar pregnancies and metastatic disease is rare. [1]

The disease is diagnosed by clinical history, physical examination, routine hematological, serological, radiological and serum $\beta$-hCG estimation. Histological evaluation is necessary for CCA that are resistant to chemotherapy. Pathologist should review the specimen carefully to avoid missing any existing intermediate trophoblastic tumor (ITT) component i.e. PSTT and ETT. Surgery is the primary treatment modality as adjuvant chemotherapy appears to provide no benefit in patients with pure ITT. GTN are highly curable in spite of metastatic disease due to high sensitivity to chemotherapeutic agents with cure rate reaching up to $100 \%$ while minimizing unnecessary excess chemotherapy 
administration thus preserving fertility. [3] GTN is subdivided into low and high risk group according to FIGO prognostic scoring system (Table 1). Serum $\beta$-hCG is a disease specific biomarker. Its estimation should be done before evacuation of mole, then weekly for three weeks and then monthly up to one year and in follow up.

FIGO anatomic Staging 2002
Stage I - Disease confined to the uterus

Stage II -GTN extends outside the uterus, but is limited to the genital structures (adnexa, vagina and

broad ligament).

Stage III - GTN with lung metastasis with or without genital tract involvement.

Stage IV - All other metastatic sites involved

Table 1. Modified World health organization (WHO) prognostic scoring system as adapted by FIGO Year 2000.

\begin{tabular}{llll}
\hline Prognostic factors & Risk Score 0 & Risk Score 1 & Risk Score 2 \\
\hline Age in years & $\leq 39$ & $\geq 40$ & Risk Score 4 \\
Antecedent pregnancy & Mole & Abortion & Term \\
Interval from index pregnancy $(\mathrm{months}) *$ & $<4$ & $4-6$ & $7-12$ \\
Pre-treatment serum $\beta$-hCG $(\mathrm{mIU} / \mathrm{mL})$ & $<1,000$ & $1,000-10,000$ & $10,000-1,00,000$ \\
Largest tumor size including uterus $(\mathrm{cm})$ & $<3$ & $3-4$ & $>5$ \\
Site of metastases & & Spleen, Kidney & Gastrointestinal Tract \\
Number of metastases identified & 0 & $1-4$ & Brain, Liver \\
Prior failed chemotherapy & - & - & Single drug \\
Total Score & Low Risk Group & Upto 6 & High risk Group \\
\hline
\end{tabular}

*An Interval time (in months) between end of antecedent pregnancy and start of chemotherapy

FIGO -International Federation of Gynecology and Obstetrics

Table 2. Level of $\beta$ - $h C G$ and duration of normal pregnancy.

\begin{tabular}{ll}
\hline Post LMP (pregnancy in week) & serum hCG $(\mathbf{m I U} / \mathbf{m L})$ \\
\hline 4 & $5-100$ \\
5 & $200-3000$ \\
6 & $10,000-80,000$ \\
$7-14$ & $90,000-5 \mathrm{lac}$ \\
$15-26$ & $5000-80000$ \\
$27-40$ & $3000-15000$ \\
Non pregnant & $<5$ \\
Trophoblastic disease & $>1$ lac \\
\hline
\end{tabular}

\section{Methods}

\subsection{Study Design}

A retrospective analytic study of 23 women diagnosed to have GTN over a period of 18 years (2002-2020) was done. The 'study' group consisted of patients diagnosed in department of obstetrics and gynecology of our institution as well as other hospitals; who were referred for further treatment to department of radiotherapy. They were evaluated and treated in the department of radiotherapy and represent cases that completed at least two years follow up. The records of these patients were evaluated for: informed written consent, age, obstetric history, type of antecedent pregnancy (abortion, molar/ term/ectopic pregnancy) and treatment received before coming to this institution. [Table 3] Patient details including general physical examination, pelvic examination, routine hematological, serological tests including serum $\beta$-hCG, USG (abdomen, pelvis and transvaginal) and skiagram of the chest were noted for clinical staging and risk performance scoring. Computerized Tomography of chest and brain were done if metastasis were suspected. WHO scoring was done to know the prognosis and treatment planning (Table 1). Low risk group patients (score six or less than six) were given injection Methotrexate and Calcium leucovorin whereas high risk group (score 7 and above) were given EMACO regimen. Serum $\beta$-hCG estimation was done every two weeks in all patients during chemotherapy treatment. Remission was considered when $\beta$ HCG levels remained within normal limits for three consecutive weeks. Then $\beta$-hCG level was done every month till the end of one year in low risk group and up to 2 years in high risk group patients. During follow up, the status of menstrual cycle during and after chemotherapy was asked and clinical examination, USG abdomen was done. Patients were advised contraception up to two years. Owing to the simultaneously loss of pregnancy and the threat of malignancy, women with GTN run a greater risk of developing severe psychological problems and should receive counseling, psychological support and interventions that may mitigate these disturbances.

\subsection{Chemotherapy Protocols}

Chemotherapy in a low-risk gestational trophoblastic tumor

Injection Methotrexate $1 \mathrm{mg} / \mathrm{kg}$ IM Day 1,3,5,7

Injection Calcium Leucovorin $0.1 \mathrm{mg} / \mathrm{kg}$ IM Day 2,4,6,8

Cycle to be repeated after 14 days

Chemotherapy in a high-risk gestational trophoblastic tumor

Inj. Etoposide $100 \mathrm{mg} / \mathrm{m}^{2} \mathrm{IV}$ - day 1,2

Inj. Methotrexate $100 \mathrm{mg} / \mathrm{m}^{2}$ IV stat - day 1

Inj. Methotrexate $200 \mathrm{mg} / \mathrm{m}^{2} \mathrm{IV}$ infusion (over12 hours) day 1

Inj. Actinomycin-D $0.5 \mathrm{mg} / \mathrm{m}^{2} \mathrm{IV}$ - day 1,2

Inj. Calcium Leucovorin $15 \mathrm{mg}$ IV/ IM BD for two days to be given 24 hours after the start of injection methotrexate.

Inj. Vincristine $1 \mathrm{mg} / \mathrm{m}^{2} \mathrm{IV}$ stat - day 8

Inj. Cyclophosphamide $600 \mathrm{mg} / \mathrm{m}^{2} \mathrm{IV}$ stat - day 8 
Injection Methotrexate (preservative free) $12.5 \mathrm{mg}$ intrathecally D8

Liquid Di Sodium Hydrogen Citrate 10cc dissolved in a glass of water three times a day for D 1-5

Injection Filgrastim 300 mcg S.C. Day 3 and 8

Cycle to be repeated after 14 days

Radiotherapy $30 \mathrm{~Gy} / 10$ fractions to whole brain over two week's period in patients with brain metastasis

\section{Results}

\subsection{Demography and Classification}

The age ranged from 20-42 years (Mean age 27.26 years) [Table 3]. Majority of patients 15 (65.21\%) were nullipara and primipara. Twelve patients were diagnosed to have invasive mole ( 11 were complete and 1 was partial mole). Nine cases were diagnosed to have choriocarcinoma. One patient developed vault recurrence after caesarean hysterectomy. One patient of ectopic pregnancy was diagnosed choriocarcinoma after histopathological examination of excised ectopic pregnancy.

The serum $\beta$-hCG level before starting chemotherapy was $>1$ lac in $10(43.47 \%)$ patients. Majority of patients 11 $(47.82 \%)$ were in stage I. The WHO scoring was 6 or $<6$ in 13 $(56.52 \%)$ and 7 or $>7$ in $10(43.47 \%)$ patients. Ovarian enlargement was found in $6(26.08 \%)$ patients on USG examination. Three patients presented with amenorrhea and acute abdomen. On laparotomy uterine perforation was found and hysterectomy was done. The duration between antecedent pregnancy and initiation of treatment was $<4$ months in 19 $(82.6 \%)$ patients.

\subsection{Treatment and Outcome}

All 23 patients were given anti-cancer chemotherapy Methotrexate + Calcium Leucovorin in $13(56.52 \%)$ and EMACO in $10(43.47 \%)$ patients. One patient presented with both pulmonary and intracranial metastasis. Another patient presented with intra cranial space occupying lesion along with bilateral pulmonary shadows and molar pregnancy. Craniotomy was done and histology revealed choriocarcinoma. She was given EMACO regimen. After 3 cycles, the $\beta$-hCG level came to a plateau. Whole brain cranial radiotherapy was given 30Gy/10 fractions to both these patients. In 11 (47.82\%) patients less than 4 cycles and in $12(52.17 \%)$ patients more than 4 cycles of chemotherapy were administered. One more chemotherapy cycle was administered after CR in all patients.

One patient showed poor response to Methotrexate + Calcium Leucovorin regimen so EMACO regimen was given. One patient was found HIV positive on routine investigation. She achieved complete remission and was lost to follow up. One patient developed pulmonary metastasis with elevated $\beta$-hCG in follow up after 11 years of treatment. EMACO regimen was given and she achieved CR. Eight women conceived after treatment and delivered healthy babies. Hysterectomy was done in 4 patients (TAHBSO- 2, subtotal hysterectomy -1 and TAHBSO with omentectomy-
1).

\subsection{Follow up}

Except for one patient who was lost to follow up, 22 are presently surviving and being followed up with annual serum $\beta$-hCG monitoring. Three (13.04\%) patients relapsed after primary chemotherapy treatment thus response rate was $87 \%$. All three patients were given second line chemotherapy and achieved CR. The patient who developed brain metastasis, conceived 3 years after treatment and delivered a healthy male child weighing $3 \mathrm{Kg}$. Thus overall cure rate is $100 \%$ Only few patients developed grade I anemia, leucopenia and neutropenia. Two patients developed oral mucositis grade II. Ten patients developed reversible alopecia.

Table 3. Clinical characteristics of patients with GTN.

\begin{tabular}{|c|c|c|c|}
\hline Age (Years) & $\begin{array}{l}\text { Mean Age } 27.26 \\
\text { (Range 20-42 Years) }\end{array}$ & Number & Percentage \\
\hline \multirow{6}{*}{ Parity } & $<20$ & 2 & $8.69 \%$ \\
\hline & $21-35$ & 19 & $82.6 \%$ \\
\hline & $>35$ & 2 & $8.69 \%$ \\
\hline & Nullipara & 7 & 30.43 \\
\hline & Primipara & 8 & 34.78 \\
\hline & Para 2 & 4 & 17.39 \\
\hline \multirow{3}{*}{ Antecedent Pregnancy } & Para 3 or more & 4 & 17.39 \\
\hline & Molar & 12 & 52.17 \\
\hline & Abortion & 9 & 39.13 \\
\hline \multirow{5}{*}{ Duration of disease } & Term & 1 & 4.34 \\
\hline & Ectopic & 1 & 4.34 \\
\hline & Months & & \\
\hline & $<4$ & 19 & 82.6 \\
\hline & $4-6$ & 3 & 13.04 \\
\hline \multirow{4}{*}{ Pre treatment B-hCG } & $>6$ & 1 & 4.34 \\
\hline & $\mathrm{mIU} / \mathrm{dl}$ & & \\
\hline & $<1000$ & 4 & 17.39 \\
\hline & $1000-10000$ & 3 & 13.04 \\
\hline \multirow{6}{*}{ FIGO Staging } & 10000-1 Lac & 6 & 26.08 \\
\hline & $>1 \mathrm{Lac}$ & 10 & 43.47 \\
\hline & Stage I & 11 & 47.82 \\
\hline & Stage II & 4 & 17.39 \\
\hline & Stage III & 6 & 26.08 \\
\hline & Stage IV & 2 & 08.69 \\
\hline \multirow[t]{2}{*}{ WHO Scoring } & 6 or less than 6 & 13 & 56.52 \\
\hline & 7 or more than 7 & 10 & 43.47 \\
\hline \multirow[t]{2}{*}{ Type of GTN } & Invasive Mole & 12 & 52.18 \\
\hline & Choriocarcinoma & 11 & 47.82 \\
\hline \multirow{4}{*}{ Treatment given } & PSTT/ETT & 00 & 00 \\
\hline & Chemotherapy & 23 & 100 \\
\hline & Hysterectomy & 4 & 17.39 \\
\hline & radiotherapy & 2 & 8.69 \\
\hline \multirow[t]{2}{*}{$\begin{array}{l}\text { No. of chemotherapy } \\
\text { cycles given }\end{array}$} & Less than 4 & 11 & 47.82 \\
\hline & More than 4 & 12 & 52.17 \\
\hline \multirow{2}{*}{$\begin{array}{l}\text { Chemotherapy } \\
\text { Protocol }\end{array}$} & Methotrexate+Calcium & 13 & 56.52 \\
\hline & EMACO & 10 & 43.47 \\
\hline
\end{tabular}

\section{Discussion}

The incidence of GTD varies from 1 in 1000 pregnancy in the West to 1 in 100 in Philippines [4]. These patients usually present with bleeding per vaginum, early pregnancy failure, hypertension or excessive uterine enlargement. [5, 6, 7] Early 
diagnosis is possible by USG and Color Doppler study. USG not only provides information on the type of molar pregnancy but also reveals involvement of endometrial cavity or myometrium and assesses the risk of the perforation, detects theca lutein cysts and also vaginal and ovarian metastasis.

The etiological factors are vitamin A deficiency, history of infertility and spontaneous abortion. Both very young women and women more than 40 years of age have higher risk of complete molar pregnancy with older women having a 5-10 fold higher risk. In women over the age of 50 years one out of every 3 pregnancies is molar. There is association of increasing uterine size with subsequent need for chemotherapy. The risk of molar pregnancy increases in subsequent pregnancies. The complications associated with uterine evacuation are hemorrhage, tumor embolization, uterine perforation and sepsis. In our study, three patients had uterine perforation. Repeat or second uterine evacuation has a role in selected cases of persistent trophoblastic disease whose $\beta$-hCG was less than $1500 \mathrm{IU} / \mathrm{ml}$, and USG showed significant molar tissue. The diagnosis was made by histopathology of evacuated material and ploidy test immunoglobin with p57 KIP 2 to confirm complete mole. [4] Introduction of effective chemotherapy has made this once fatal malignancy curable. For low risk GTN - Methotrexate + Calcium Leucovorin regimen is advocated and in high risk GTN, EMACO regimen is found to be appropriate. In relapsed low risk GTN patients previously treated with Methotrexate +Calcium Leucovorin, EMACO regimen should be considered.

Patients with widespread metastatic GTN, with a prognostic score $>12$, have a poorer prognosis. [8] Initiation of standard combination chemotherapy in these patients can lead to tumor lysis syndrome resulting in high morbidity and mortality. [8, 9] Bolze et al in a case series of 140 patients with high-risk GTN, reported 33 patients with large disease burden who received low-dose induction chemotherapy with etoposide / cisplatin before EMA/CO therapy (etoposide 100 $\mathrm{mg} / \mathrm{m}^{2}$ and cisplatin $20 \mathrm{mg} / \mathrm{m}^{2}$ on days 1 and 2, every 7 days for 1-3 courses). [8] Overall survival and early death rate were $94.3 \%$ and $0.7 \%$, respectively.

PSTT and ETT are extremely rare forms of malignant GTN and there are insufficient data to characterize the epidemiology or risk factors. About $95 \%$ of PSTT and ESS lesions develop after a term pregnancy or non molar abortion, often several months or even years later. Indeed the management of PSTT and ETT is quite different, reflecting its distinct clinical behavior. The disease is slow growing, produces less serum $\beta$-hCG; remains confined to the uterus for longer time, is more likely to involve regional lymph nodes and is a little more resistant to chemotherapy than CCA. The patients with histologically confirmed PSTT/ETT confined to the uterus should undergo hysterectomy whilst those with metastatic disease need combination agent chemotherapy followed by resection of residual disease. [1]

The recurrence of GTN in subsequent pregnancy being 1 in 60 after 1 st molar pregnancy, 1 in 10 after $2^{\text {nd }}$ molar pregnancy and 1 in 2 after $3^{\text {rd }}$ molar pregnancy. The recurrence is usually of the same histopathological type. Bower el al - studied 112 live births following chemotherapy and found 3 infants with congenital anomalies. They stated that the risk of congenital anomalies in infants born to mothers treated with all forms of chemotherapy for GTN is $0.8-3.4 \%$. [10]

During subsequent pregnancies in treated women, the placenta should be sent for histopathology and serum $\beta$-hCG should be evaluated at the end of puerperium i.e. 6 weeks post partum. The overall survival rates for patients with high risk group are now as high as $95 \%$ while in low risk group, CR rate is close to $100 \%$.

Despite the use of multi agent primary chemotherapy, approximately $30 \%$ to $40 \%$ of patients having high risk score will have an incomplete response to first-line therapy or will experience relapse. [11] Most of these patients have multiple metastases to sites other than the lung and vagina and many will have received inadequate initial therapy. [12]

The EMA/EP or EP/EMA regimens are considered the most appropriate therapy for patients who have either partial response to or have relapsed after EMACO regimen. [13] The rate of complete response in such patients is reported between $75-85 \%$. [13-14]

Additional drug combinations containing etoposide and a platinum agent have been effective in patients who have developed disease resistant to methotrexate-containing regimens. These include TP/TE (paclitaxel and cisplatin alternating weekly with paclitaxel and etoposide), BEP (bleomycin, etoposide, and cisplatin), VIP (etoposide, ifosfamide, and cisplatin) and ICE (ifosfamide, carboplatin, and etoposide) [15-17]. The TIP regimen (paclitaxel, ifosfamide, and cisplatin) has been used as a salvage chemotherapy in germ cell tumors, including those with choriocarcinoma components. [18] These etoposide-platinum containing regimens require the use of granulocyte colonystimulating factor support to prevent neutropenic complications and treatment delays. [5, 19] The overall success of salvage therapy in this group of patients is about $80 \%$. Factors associated with worse survival outcomes include high $\beta$-hCG at the start of salvage therapy, greater number of metastatic sites, metastases to sites other than the lung and vagina (stage IV) and FIGO score $>12$.

High dose chemotherapy (HDC) with autologous stem cell support has been reported to produce sustained complete responses. [20] A retrospective study of 32 patients with refractory choriocarcinoma or poor-prognosis PSTT/ETT who underwent HDC with peripheral blood stem cell support reported a sustained complete response in 7 patients. [20] Pembrolizumab is a monoclonal antibody that inhibits programmed cell death protein 1 (PD-1), which functions as a check point protein for regulation of various immune cells, including $\mathrm{T}$ cells with potential antitumor activity. [21] Programmed death ligand 1 (PD-L1) is strongly expressed by GTN. [22] Outcomes were recently reported for 4 patients with drug-resistant GTN who received pembrolizumab, including 2 with metastatic choriocarcinoma and 2 with 
metastatic PSTT or mixed PSTT/ETT. [23] All patients had tumors with high levels of PD-L1 expression. Durable response to pembrolizumab was seen in 3 of the 4 patients. The patient whose disease did not respond to pembrolizumab had strong PD-L1 tumor expression but an absence of tumorinfiltrating lymphocytes. [23] Gemcitabine, capecitabine, and fluorouracil may also have potential for treating GTN in this setting.

\section{Conclusion}

Early diagnosis and adequate treatment ensures excellent prognosis in these patients. The FIGO/WHO scoring system (2000) has provided a universal language for the discussion of this neoplasia and has been used to define prognostic factors more accurately and to compare different treatment protocols for low and high risk GTN. The serum $\beta$-hCG level has a key role in the diagnosis, treatment and follow up of GTN. The patients with GTN should be treated by a tertiary care center where they will receive specialized care which has direct impact on survival and quality of life. Introduction of effective chemotherapy has made this once fatal malignancy curable. Methotrexate and Calcium Leucovorin is highly effective chemotherapy for low risk group whereas EMACO regimen is best option in high risk group. We found that treatment was highly effective without any serious adverse effects. Any pregnancy should be followed up with $\beta$-hCG values at 6 weeks post partum. The importance of contraception and follow up should be emphasized to patients. Furthermore spreading awareness in Indian masses about this rare but chemo responsive malignancy with high response rate and high fertility rate may help in reducing the number of women lost to follow up. We acknowledge that this retrospective study represented a single center experience with best results at par with any other centre of excellence.

\section{Conflicts of Interest}

None

\section{References}

[1] Berkowitz RS and Goldstein DP. Molar pregnancy. N. Engl. J. Medicine 2009; 1639-45.

[2] Mangili G, Lorusso D, Brown J,. Trophoblastic disease review for diagnosis and management: a joint report from the International Society for the Study of Trophoblastic Disease, European Organisation for the Treatment of Trophoblastic Disease, and the Gynecologic Cancer Intergroup. Int J Gynecol Cancer 2014; 24 (9, Suppl 3) S109-S116.

[3] Tse KY, Chan KKL, Tam KF and Ngan HYS. Gestational trophoblastic disease. Obstetrics Gynecology and Reproductive Medicine. 2009; 19 (4): 89-91.

[4] Savage P. Molar pregnancy. The Obstetrician and Gynecologist 2008; 10: 3-8.
[5] Lurain JR. Gestational trophoblastic disease I: epidemiology, pathology, clinical presentation and diagnosis of gestational trophoblastic disease, and management of hydatidiform mole. Am J Obstet Gynecol 2010; 203: 531-539.

[6] Seckl MJ, Sebire NJ, Berkowitz RS. Gestational trophoblastic disease. Lancet 2010; 376: 717-729.

[7] Brown J, Naumann RW, Seckl MJ, 15 years of progress in gestational trophoblastic disease: scoring, standardization, and salvage. Gynecol Oncol 2017; 144: 200-207.

[8] Bolze PA, Riedl C, Massardier J,. Mortality rate of gestational trophoblastic neoplasia with a FIGO score of $\geq 13$. Am J Obstet Gynecol 2016; 214: 390. e391-398.

[9] Ngan HYS, Seckl MJ, Berkowitz RS, Update on the diagnosis and management of gestational trophoblastic disease. Int $\mathrm{J}$ Gynaecol Obstet 2018; 143 (Suppl 2): 79-85.

[10] Bower M, Newlands ES, Holden L Short D, Brock C, Rustin $\mathrm{CJ}$ et al. EMA/CO for high risk gestational trophoblastic tumor: Results from a cohort of 272 patients. Journal Clinical Oncology 1997; 15: 2636-43.

[11] Hoekstra AV, Lurain JR, Rademaker AW, Gestational trophoblastic neoplasia: treatment outcomes. Obstet Gynecol 2008; 112: 251-258.

[12] Lurain JR, Casanova LA, Miller DS,. Prognostic factors in gestational trophoblastic tumors: a proposed new scoring system based on multivariate analysis. Am J Obstet Gynecol 1991; 164: 611-616.

[13] Mao Y, Wan X, Lv W, Xie X. Relapsed or refractory gestational trophoblastic neoplasia treated with the etoposide and cisplatin/etoposide, methotrexate, and actinomycin D (EPEMA) regimen. Int J Gynaecol Obstet 2007; 98: 44-47.

[14] Lu WG, Ye F, Shen YM, EMA-CO chemotherapy for highrisk gestational trophoblastic neoplasia: a clinical analysis of 54 patients. Int J Gynecol Cancer 2008; 18: 357-362.

[15] Alazzam M, Tidy J, Osborne R,. Chemotherapy for resistant or recurrent gestational trophoblastic neoplasia. Cochrane Database Syst Rev 2016; 1: CD008891.

[16] Lurain JR, Schink JC. Importance of salvage therapy in the management of high-risk gestational trophoblastic neoplasia. J Reprod Med 2012; 57: 219-224.

[17] Essel KG, Bruegl A, Gershenson DM,. Salvage chemotherapy for gestational trophoblastic neoplasia: utility or futility? Gynecol Oncol 2017; 146: 74-80.

[18] Feldman DR, Hu J, Dorff TB, Lim K, Patil S, Woo KM et al. Paclitaxel, ifosfamide, and cisplatin efficacy for first-line treatment of patients with intermediate- or poor-risk germ cell tumors. J Clin Oncol 2016; 34 (21): 2478-2483.

[19] Lurain JR, Nejad B. Secondary chemotherapy for high-risk gestational trophoblastic neoplasia. Gynecol Oncol 2005; 97: 618-623.

[20] Yamamoto E, Niimi K, Fujikake K, Nishida T, Murata M, Mitsuma A et al. High-dose chemotherapy with autologous peripheral blood stem cell transplantation for choriocarcinoma: a case report and literature review. Mol Clin Oncol 2016; 5 (5): 660-664.

[21] Wherry EJ. T cell exhaustion. Nat Immunol 2011; 12: 492 499. 
[22] Veras E, Kurman RJ, Wang TL,. PD-L1 expression in human placentas and gestational trophoblastic diseases. Int J Gynecol Pathol 2017; 36: 146-153.
[23] Ghorani E, Kaur B, Fisher RA,. Pembrolizumab is effective for drug-resistant gestational trophoblastic neoplasia. Lancet 2017; 390: 2343-2345. 